\title{
INTIMACY IN THE TIME OF COVID-19
}

\author{
Jamie Foster Campbell and Zizi Papacharissi ${ }^{\mathrm{a}}$
}

\begin{abstract}
In this essay, we tell a story with data about how our relationship with technology has transformed our collective notion of intimacy and rituals of connecting with others. Framed by the COVID-19 pandemic, our starting point is that technology is neither good, bad, nor neutral. The goal of this study is to step back and have conversations with people -from all over the United States of various ages, occupations, and relationship statuses- about how they define intimacy and understand these practices in a mediated environment. In this essay, we discuss technology as more than a performative platform. It is an ambient architecture that sets the tone for interactions meant to preserve social ties and sustain social capital potentially depleted through pandemic conditions of seclusion. Through the voices of our participants, we conceptualize how intimacy is experienced and understood, and how this relational quality is amplified in a mediated environment during moments of disruption. In the end, what COVID-19 has revealed as we reflect on our relationship with technology is the fluidity of intimacy in moments of change.
\end{abstract}

Keywords: intimacy; media; COVID-19.

${ }^{a}$ University of Illinois at Chicago, United States. 
In the acclaimed novel Love in the Time of Cholera, Gabriel García Márquez seductively deconstructs myths about love, passion, and immortality through a powerful narrative. ${ }^{1}$ The pandemic serves as both context and metaphor, against which the characters latch on, reimagine, or abandon their mythology of being. We take inspiration from the magical vernacular Márquez offers. Through interviews with everyday strangers, ${ }^{2}$ all ensconced in their private islands of community and isolation, we can revisit the meaning of intimacy in the time of our current pandemic. As technology, for many, scales from a supplemental to a primary avenue for connection and expression, how does our psyche, collective and personal, adjust to this transition?

The data presented in this essay originate from a larger qualitative project that focuses on how intimacy is mediated through communication technologies. The goal of this research is to step back and have conversations with people - from all over the United States of various ages, occupations, and relationship statuses - about how they define intimacy and understand these practices in a mediated environment. In the end, this research is about uncovering how mobile technologies have transformed our collective notion of intimacy and the rituals of connecting with others. What we hope to provide here is a snapshot of the stories our informants shared and our analysis of the larger research question guiding this investigation: What pressure does COVID-19 put on the relationship between technology and intimacy?

Our experiences with COVID-19, as a society, present a unique lens through which to re-examine what intimacy means, how it is experienced during a pandemic, and what role technology plays in this equation. For instance, Oliver remarks that "Having technology makes me feel like I am part of a community all the time" and goes on to explain that he views technology as "a tool for livelihood." 3 Technology shapes our perception of social and relational life. We often take for granted how quickly media become part of the social fabric of our lives, and this invisible infrastructure frames our daily interactions. For many of us during the pandemic, we found ourselves living our social lives primarily through a screen. These invisible, everyday technologies, became the center of our way of life.

\footnotetext{
${ }^{1}$ Márquez, G. G. (1997). Love in the time of cholera. Everyman's Library.

${ }^{2}$ The interviews talked about in this piece were conducted by Jamie Foster Campbell as part of her dissertation research under the mentorship of Dr. Zizi Papacharissi. The accounts represented here are an illustration of the data collected between May 2020 and December 2020.

${ }^{3}$ The quotes referenced here come from people who are 23-64 years old and identify as straight, gay, or queer, whose occupations vary (e.g., Editor, Tattoo Artist, RN, Early Childhood Educator, Office Manager, Social Worker), and locations include California, Washington, Oregon, Colorado, Kansas, Illinois, and Georgia.
} 
Gabriella adds, "Before the quarantine, there was a period of maybe 5 months I was home alone with the baby and I think I was more socially isolated during that time than I have been since the quarantine and that is because everyone is isolated at home, not just me." Gabriella says this laughing, but the reality is as a first-time mom she has felt less isolated since the pandemic started because she can now join in on conversations that she would normally miss, whether that be during a Zoom happy hour or when she attends virtual events with her family and friends. In this case, Gabriella is reimagining intimacy in her relationships by engaging in different ways that make her feel more connected.

Intimacy is dynamic and relational; experienced not just in the context of our everyday relations, but also measured against the backdrop of how we think others experience intimacy. We, as a society, often worry that social media increases our fear of missing out; it would appear that technology can also flatten that fear; sometimes diminishing it and sometimes rendering it stable. To this point, Sophie adds, "my communication with my adult children is more constant right now, so you get a glimpse into the small moments of their daily life. If you wait for a couple of weeks to talk or for the next time you're all together you miss things... we only talk about the bigger moments. But now since we are communicating more through technology I get to hear about the daily frustrations or struggles, the triumphs and it helps me understand a little better and feel more connected to them."

Technology is a shared cultural backdrop and a place for communication. It lends a space for interaction; a way to extend feelings of intimacy with close relational others as we traverse through our worlds. We are conditioned to think of technology as an alternative path to intimacy, pursued only in the absence of other, preferred routes. Yet we end up using technology for intimacy more often than we realize in our everyday lives, and even more so now, from our sheltered abodes. How we envision and practice intimacy in our close relationships is formed by what we expect technology to offer; and what we are sure it cannot. We apply these expectations to our design of technology, inadvertently limiting the true potential of what technologies might provide. Still, technology is woven into the daily fabric of our interactions, our intimacy vocabulary, our relational histories, and our sense of being. Technology, in its many forms and platforms, supports our soft structures of intimacy.

Intimate acts are momentary, lasting for a brief time - however, they are also everywhere and necessary for social connection. "Intimacy, for me..." says Marie, "is feeling really seen. Like in a way that somebody can only do if they really know you on a very deep level. It is feeling safe with somebody and very connected." Marie highlights how intimacy is not just associated with romantic love or physical acts but related to trust, 
understanding, and exposure. This same sentiment is expressed by Marcos who describes intimacy as layered, he says "I tend not to think of physical intimacy as the end all be all because I don't hold physical intimacy on this pedestal, this pure thing that needs to be sacred. I would much quicker qualify my in-depth conversations with my best friends as intimate than I would me sexting a random guy that I just met and who will ghost me in a week. While that conversation with the guy could be seen as intimate, I don't consider it as intimate because there is no emotional context there." Thus, intimacy is linked to emotion, it is fueled by time, and grows alongside our relationships. Intimate acts and the feeling of intimacy are not always viewed as the same construct among our informants. Intimacy can also be experienced in the fleeting moment, between passing strangers who feel seen as they evanescently cross paths. Gabriella believes intimacy is "space, either physically or intellectually, where you are actively expressing and receiving without any judgment... It's an act without consciousness." So, intimacy is not necessarily tangible or something we all define equally. Intimacy frequently lurks in the background and often manifests itself in invisible ways, that is, moments that we label intimate upon further reflection.

Thus, temporality not only punctuates our acts of intimacy. It defines how we interpret them. Nora passionately says "Intimacy is time. Personal time and that's it. Time is the greatest luxury. Time is the biggest gift we can give someone, our focused attention, our singular attention. Slowing down. Taking the time to make time, to be present, to be available. To me, that is intimacy." As we retreat to our homes, work from our living rooms, and have more time to talk to our close networks through our screens we see how intimacy is subjectively experienced. As technology becomes the mediator for social gatherings, and the pandemic surges on, time acquires a different meaning. We have more time to reflect on how we use technology to cultivate intimacy. We have less time to manage the new insecurities brought on by the pandemic. Yet the benefit of time often affords reflection and hindsight, in overcoming insecurities and as we craft the ties of intimacy. Time shrinks and expands simultaneously, and our technologies permit us to both stretch and speed up time. Yet the platforms we use frequently suggest ways of doing that limit our options. They afford ways of transferring our routines online, and this transition is uneasy. Different spaces produce varying paths to intimacy. We are speeding down the desire lines of intimacy as we move our gatherings online. In our efforts to adjust, we turn to the familiar and seek to transition that online. Desire paths in nature typically reflected the most often travelled route. Still what worked for us pre-pandemic may not meet our pandemic wants. We change and evolve during the pandemic, and we will possible return to a normal, post pandemic, that we no longer recognize as familiar. When we go back 
to what was normal, we will no longer be the same people who live in that normal.

We quickly learn how to use technology to bend time as we live through times that feel simultaneously fast and painfully slow. We can't catch the eye of someone across the room, so Orion explains how he is 'dating' differently through his smartphone now: "I've got the chance to know more about people because of this...I can't see you, so therefore I can't touch you, so we are having conversations. I get to sit down and actually talk to them, get to know them, it's different." Orion speaks about how before he would call someone, they would get together, and it was physically driven. He then explains, “... now I actually get to know people. It's like 'oh wow... is that a Bears picture in your background, you're into football, we can watch a game together.' I am finding out a lot about people that I would have never bothered to ask."

Our collective experience with COVID-19 provided opportunities that would not have existed outside of this moment. For instance, Lola explains how she initially met her 74-year-old neighbor through a community Facebook group when her neighbor posted a question asking for a house cleaner recommendation. "We ended up chatting on messenger in that way," said Lola, "it was at the beginning of the year, right around the time of the pandemic and I realized I don't know if anyone's checked on her? Does she need groceries? So I sent her a text message and she replied 'I'm very depressed and very scared' so I said 'why don't we do a social distancing happy hour and we'll have some wine.' This was the first time we met in-person, and we've been doing it every Friday since then." Lola continues to explain that this ritual has continued throughout the pandemic and she's formed a close friendship with her neighbor, which all started from a group message on social media. "We both look forward to this every week" explained, Lola. "It's also during a time where we need things to look forward to. And also for her, it's something to do that gets her some human interaction."

In these stories we see how moments carved out in the time of COVID19 are chances for intimacy to be experienced in big, small, new, and reconstructed ways. Technology provides opportunities for us to experiment with intimacy and forge renewed possibilities to connect with others. Grace describes how her increased use of Zoom, and other video applications to talk to friends and family, helps her feel more in tune with people and catch up with those she usually only gets to see once or twice a year. Grace said "we've probably shared more in the past 3 months than we would have over the course of 5 years. I am forced to trust technology a little more now and I really do believe that technology enhances our relationships, it bonds us." 
Still, stories of connection surface among our informants as often as stories of fatigue with technology. For instance, Patrick explains that "the longer this goes on, I think it almost draws more attention to the fact that things aren't normal. For me, it doesn't make me feel better, it brings into focus the gravity of the situation. This is what we're forced to do... and everything with technology now is kind of a reminder that our 'normal' lives will never return the same way." Lucy also continues to talk about the reality that is setting in as the pandemic continues. When we spoke in May of 2020 she said, "Now, the more I engage in video chatting the harder it's becoming. I get off the call and I am sad because I realize even more now that my physically near and physically distant relationships are happening solely through my laptop. So, eight weeks in... I am experiencing more points of grief; the mediated interactions are starting to drain me now."

Iris adds that one of the biggest lessons she's learned from the pandemic is that "technology is super limiting. It is great for being able to share small snippets of your life and it is great for having a conversation but [long pause] it will be nice when I can visit and see people again, it does not actually resolve that desire." Iris explains how technology is replacing social gatherings during COVID-19 and that is just the reality of our current situation, but the more she uses technology to talk to people, engage in virtual experiences together, seek information, and so on, the more she realizes what we cannot do through this medium. As we talk she laughs and says "you can't share food virtually" - for someone that cultivates intimacy through sharing food and cooking for others this is what she misses most. Iris said, "We can have a painting party virtually, but we can't eat the same cheese together on a piece of bread."

Through these interviews, we've learned that intimacy is an abstract, yet familiar term. Intimacy is dynamic; it happens in a lot of different places and with a lot of different people, as well as relationship types. In the age of COVID-19, we are becoming creative and intentional with how we use technology because media are the primary paths to intimacy. So, what does intimacy look like during a pandemic? We think it is still the act of sharing, supporting, and revealing the raw parts of ourselves to others. It is a reminder that we are a part of a community. It evolves beyond romantic love gestures or physical acts of connection. Intimacy is a visceral feeling. It can be sustained through technology. Intimacy and connection are imbricated across the different layers of communication technologies we, as a collective, use daily.

When asked what the future holds, Bella quickly offered that, "there are certain things - like virtual happy hours or movie parties that I would like to carry on since I live in a different city now than my community. I've been communicating more with friends and family that don't live nearby and I really like that." Yet Patrick countered that, "when we return to some state 
of being together offline I don't think we will realize how much things have changed until it is written in the history books." Andrew reminds us "there's no replacement for personal connection, physical in-person interaction. There's no replacement for that and I think people are doing the best that they can." Luciana agrees that we cannot expect technology to replace the in-person interactions, but "we're so fortunate to have this technology" she explains, with her increased use of video calls for work and her social life right now she realizes that "it's such a different experience talking to people where you can see them, their body, their rooms and expressions, all of that, it adds to the intimacy, the connection between you and whoever you're talking to." No one has a crystal ball that allows one to look into the future. Our informants suggest that some of these rituals will stay and some will disappear. Importantly, as our informants also reveal, time will allow us to understand how this has affected us, as a collective, with greater clarity.

Indeed, a temporal paradox resurfaces as conversations on intimacy focus on the COVID condition. We are asked to adjust our routines during a time where the odds change daily, if not hourly, and insecurity is heightened. Slow living at a fast pace is the dominant ethic of our time. Our technologies, including our platforms, our media, our hardware but also our homes, our offices, our streets were not designed for slow living. Technology is our architecture of many forms, and it is an architecture that was designed to accommodate acceleration, multi-tasking, and a culture of expedition. Our primary platforms support transient interaction across the globe, not intimacy from a distance. Still, we find ourselves in and give ourselves comfort through structures of feeling and solidarity, facilitated through social media.

In Chicago, our home base, we used platforms to coordinate new routines of blinking our lights, honking our horns, signing and clapping together, banging surfaces to the rhythm of social solidarity. We told ourselves that we were applauding health workers, and we were, but we were also patting ourselves on the back and signaling intimacy across our balconies, windows, and spheres of solitude. We were seeking ways of performing slow living amid cities, suburbs, towns, structures, and technology that support the accelerated rhythms of times past. The pandemic is inviting us to rethink how we, as a society, understand intimacy, much like disease (cholera, in Gabriel Garcia Marquez's case) prompted previous generations to think again about love and modes of being.

So, this is not 'new' to the era of COVID-19. Communication technologies of the past have played a significant role in growing the seeds of intimacy in close relationships when distance and pandemics separated loved ones. For instance, letters have long been associated with intimacy, 
viewed as a private space to communicate with close ties. ${ }^{4}$ Writing - on a papyrus scroll, in sealed letters, through telegraph wires, or smartphone keyboards - is a way for messages to travel, and intimacy to unfold and live on as an archive of people's experiences and imagination. Writing promotes rituals of expression and discovery; and has long been a part of our culture of communication. With each of these technologies, from handwritten letters to telephone conversations, comes a new culture of communication that changes our habits and the way we think. Communication technologies are woven into the many layers of human behavior over time and just like with other historical pandemics, people have needed to distance themselves from their communities before. Thus relying on the technologies at hand to feel connected to others as they imagine their prose, hear their voice, or now see their face through a screen.

People have been intimate in moments of chaos before. People still love; they are intimate in physical, emotional, and intellectual ways. People start and end relationships, as well as experience major life and relational transitions - these do not stop because our familiar ways of life are disrupted. What COVID-19 has revealed as we reflect on our relationship with technology is the fluidity of intimacy in moments of change. The mediated nature of intimacy that is amplified by pandemic conditions. Our desire to be seen, heard, understood remains. Our technologies feast on this want and this desire. One is reminded of the prescient words of Alluquère Rosanne (Sandy) Stone (1995) well before the close of the previous century, in The War of Desire and Technology at the Close of the Mechanical Age:

Electronic virtual communities represent flexible, lively, and practical adaptations to the real circumstances that confront persons seeking community ... They are part of a range of innovative solutions to the drive for sociality - a drive that can be frequently thwarted by the geographical and cultural realities of cities increasingly structured according to the needs of powerful economic interests rather than in ways that encourage and facilitate habitation and social interaction in the urban context. In this context, electronic virtual communities are complex and ingenious strategies for survival..$^{5}$

Different worlds lurk behind the screen and our collective experience during this COVID crisis has magnified the liquid nature of intimacy, and the role technology plays in our relational lives as we strive for well-being

\footnotetext{
${ }^{4}$ For a deeper discussion on the intimate nature of letter writing and embodied presence see Milne, E. (2003). Email and epistolary technologies: Presence, intimacy, and disembodiment. Fibreculture, 2(2), 1-14.

${ }^{5}$ Stone, A. R. (1995). The war of desire and technology at the close of the mechanical age. MIT press.
} 
in moments of disruption. ${ }^{6}$ These stories show us that intimacy is both mundane and unique, it is necessary for the human connection and something we build in ways that feel familiar and novel at the same time.

Ultimately, as the pandemic surges on we see how intimacy manifests itself in the place of technology by allowing us to share experiences and provide support. For instance, our communication devices enable us to send text messages, memes, share articles and resources, or video chat with our network. They provide a space for us to hold events virtually like a book club, concerts, or dinner parties. COVID-19 forced us to think about our social connections and intimacy differently, and in response, we are also creating new rituals for how to navigate this world. We are experimenting with how to use the technology that serves our lives right now, investing our time and energy into media that acts as a bridge between ourselves and our social networks. We are living with and through these technologies in a way that allows us to reevaluate what we want from our social connections, and to consider how technology is transforming our experiences of intimacy.

One thing is clear based on the research referenced above: We all understand intimacy in different ways, but we will use everything and anything at hand, and will creatively adapt it as we strive for intimacy. The question that remains is: Have we built a society that can support our need for intimacy, through times good and bad, slow and fast, through technology and in its absence?

\section{FUNDING STATEMENT}

No funding was used as part of the research for this article.

\section{REFERENCES}

Márquez, G. G. (1997). Love in the time of cholera. Everyman's Library. Stone, A. R. (1995). The war of desire and technology at the close of the mechanical age. MIT press.

\footnotetext{
${ }^{6}$ We take inspiration from Zygmunt Bauman with the term "liquid" here. See Zygmunt, B. (2000). Liquid modernity. Polity Press.
} 